\title{
I "Peli" della Gatta Cenerentola di De Simone \\ E DEL CUNTO DE LI CUNTI DI BASILE. \\ Una PAROdia dell'illustre don BenedetTo Croce
}

\section{Pasquale Sabbatino}

\begin{abstract}
Riassunto: Nel corso del presente intervento si pone l'attenzione sul profondo e prolifico legame letterario che intercorre tra il drammaturgo novecentesco Roberto De Simone e lo scrittore seicentesco Giovanbattista Basile. In questo senso si evidenziano tre tappe salienti. De Simone debuttò nel 1976 al Festival dei due Mondi di Spoleto con un'opera intitolata La gatta cenerentola chiaramente inspirata alla favola di Basile, nel 1989 pubblico la traduzione dall'antico napoletano in dialetto moderno della raccolta Il Pentamerone ovvero Lo cunto de li cunti trattenimento de piccerille (Napoli, ed. Il Mattino), mentre del 2002 è la doppia riscrittura, in dialetto e in italiano, del Cunto de li cunti di Basile (Torino, Einaudi). L'analisi, in particolar modo, di quest'ultima opera permette, inoltre, di sottolineare il forte intento parodico di De Simone nei confronti di Benedetto Croce. Nell'anno del cinquantesimo anniversario della morte del filosofo abruzzese, De Simone decide di inserire nella propria riscrittura del Cunto di Basile il personaggio Croce, condannandolo irriverentemente a "un peteggiare involontario, ereditato dall' aerofagia patita prima di morire".
\end{abstract}

La continua ricerca di Roberto de Simone sulla cultura teatrale napoletana e sulla espressività dei linguaggi popolari si è incrociata costantemente e volutamente con Lo cunto de li cunti di Giambattista Basile, venerato come il padre del dialetto napoletano insieme a Pietro Trinchera. Non a caso, nella dedica del testo teatrale L'opera buffa del giovedì santo, De Simone scrive:

A Giambattista Basile e a Pietro Trinchera, Dante e Boccaccio (con rispetto parlando) della nobilissima lingua napoletana iuta all'acito.

E la dedica contiene una preziosa tessera utile per ricostruire, dal punto di vista di De Simone, la storia della lingua napoletana, oramai "iuta all'acito".

Nel delineare sul piano cronologico il fecondo rapporto con Basile si possono individuare tre tappe salienti: a) La gatta cenerentola (debuttò nel 1976 al Festival dei due Mondi di Spoleto); b) la traduzione dall'antico 
napoletano in dialetto moderno della raccolta Il Pentamerone ovvero Lo cunto de li cunti trattenimento de piccerille (1989), accompagnata dalla preziosa Nota illustrativa del traduttore, c) la doppia riscrittura, in dialetto $\mathrm{e}$ in italiano, del Cunto de li cunti (2002). In quest'ultima impresa, il riscrittore De Simone, appropriandosi degli strumenti narrativi del Basile, scrive in proprio un cunto che fa da Praefatio.

\section{La nuova Cenerentola e il popolo di Napoli}

Negli anni Settanta del Novecento, a Napoli e in Italia prende corpo la reazione al teatro eduardiano ${ }^{1}$. In questo ambito va collocata La gatta Cenerentola di Roberto De Simone, un singolare testo teatrale che ha la struttura di un cunto de li cunti sul personaggio della gatta Cenerentola.

Con grande abilità, sulle orme di Basile, De Simone costruisce il cunto unitario e portante di Cenerentola raccontando i diversi cunti raccolti in Campania e prelevando di volta in volta singolari e differenzianti tessere narrative. Il cunto principale ha un movimento circolare, per cui il punto di partenza coincide con il traguardo e la didascalia di apertura, che addita "il buio completo" 2 , combacia con la didascalia di chiusura, che segnala il ritorno al buio ("la luce scende fino al buio. Sipario")3. La macchina teatrale è di nuovo pronta per la ciclica rappresentazione del cunto de li cunti sulla gatta Cenerentola.

In quest'opera teatrale De Simone fa confluire innanzitutto la sesta favola della giornata prima del Pentamerone (1636) di Basile ${ }^{4}$, che costituisce il primo testo scritto della favola, divenuto fonte di Perrault e di tanti altri scrittori. Inoltre De Simone estrapola materiali narrativi dalla versione di Boccia al Mauro in provincia di Napoli (in particolare l'identificazione del luogo e del giorno della festa, cioè la chiesa e la domenica)'s, dalla versione irpina (in particolare i tre splendidi abiti), dalla versione di Briano, in provincia di Caserta (in particolare il motivo dei fiori magici, ai quali Cenerentola ripete la formula "sciure! Spogliate a te e viesteme a me!", dei

${ }^{1}$ Cfr. De Simone, "Linguaggio e tradizione nel teatro di Eduardo", 97-154; Di Stefano, "Ma ora liberiamoci di Eduardo".

${ }^{2}$ De Simone, La gatta Cenerentola, 5 .

3 De Simone, La gatta Cenerentola, 108.

${ }^{4}$ In appendice a La gatta Cenerentola, 116-118, De Simone sintetizza il cunto di Basile. In edizioni più recenti l'appendice diviene antologica, per cui De Simone riporta la trascrizione e la traduzione del cunto.

${ }^{5}$ De Simone, La gatta Cenerentola, 118. 
tre abiti, dell'andata in chiesa per ascoltare la Messa di domenica) ${ }^{6}$, e dalla versione largamente diffusa tra i pescatori di Mergellina, secondo la quale la Madonna aveva smarrito una pianella sulla spiaggia di Napoli e il ritrovamento della pianella da parte di un pescatore portò a scoprire una statua mariana nella grotta di Posillipo?

Il Prologo, recitato dal capocomico in "un vecchio teatro", come si legge nella didascalia, ci consegna il punto di vista di De Simone sull'aspetto linguistico e sull'aspetto socio-politico della Gatta cenerentola. Innanzitutto il Prologo contiene un attacco parodico e feroce sia contro ogni persona colta ("ogni alletterato 'struito") che per sfogare la propria cultura secondo la moda, "crede de have' scuperto ll'America 'nteressannose a li ccose de casa nosta", sia contro quelli che, nel raccogliere le parole napoletane solo dalla cesta dello straccivendolo, "se credono ca ogni èvera è bbona pe' la 'nzalata senza sape' addistinguere la lattuca nuvella da ll'èvera ca serve p'annettarse lu culo" 8 . I lettori e il pubblico, ai quali il capocomico si rivolge con un accattivante "Bene mio", che inaugura il gioco della complicità, si trovano di fronte a una "sferrata" contro la presunzione dei dotti, a una "bbona smerdiata" della "prusupupea de lo munno e de la gente ca se crede 'struita e ca invece va a biento comm'a li stronze sereticce 'ncopp'a lu mare"?

La nuova Cenerentola (il popolo di Napoli), secondo il De Simone degli anni Settanta, non può sperare che siano certi intellettuali - i moralisti spara-sentenza e i falsi Pulcinella dai discorsi convenzionali - a condurla in Palazzo reale e a incoronarla regina, anzi sono proprio loro a tenerla incatenata al focolaio, cioè sottomessa al potere maschile:

Napule e chella ca è, e nun sarranno certo li sparate de cierti pagliette caca-sentenze o li tirate de cierti faveze Pulicenielle ca a ttriato l'avisse sulo da sputa' 'mmocca, a cagnare la situazione. Nun sarranno li verrizze ncrapicciate de cierti pernacchie lecca-popolo ca invece stanno cu li pacche 'ncarrozza, a ffa' quacche cosa de bbuono. Nun sarranno lloro a da' tre nummere juste pe' la ruota de Napule e coserle, comme pe' sta gatta

${ }^{6}$ De Simone, La gatta Cenerentola, 119-120. Inoltre De Simone mette a confronto Basile e le due versioni (Boccia al Mauro, Villa di Briano), indicando i punti in comune e quelli differenzianti (De Simone, La gatta Cenerentola, 121-122).

${ }^{7}$ De Simone, La gatta Cenerentola, 123.

${ }^{8}$ De Simone, La gatta Cenerentola, 13.

${ }^{9}$ De Simone, La gatta Cenerentola, 14. 
Cenerentola, lu vestito pe' farla trasi” da riggina a palazzo riale. Se po' dicere anze ca chiste, aunite cu chille ca mogneno, peggio de la gnora matreia ca se la guverna tra la cennere, spisso nun fann'auto ca tenerla 'ncatenata a lu fucularo chhiú de primma ${ }^{10}$.

E nell'opera di De Simone il potere femminile di Cenerentola (ovvero il potere del popolo) è in antitesi al potere maschile (ovvero il potere sul popolo). E se il potere maschile è il potere "della guerra, della violenza e della repressione, di cui tutti i popoli hanno sempre dovuto subire l'amara esperienza" 11 , il potere di Cenerentola è il potere della pace, della ragione, della liberazione.

Così, grazie al Prologo, il teatro di Napoli diviene teatro del mondo e la conquista del potere di Cenerentola a Napoli diviene simbolo della conquista del potere di Cenerentola nel mondo, con cui inizia l'era della pace e della ragione.

\section{La traduzione in napoletano moderno del Pentamerone}

Nella dedica della traduzione dall'antico napoletano al dialetto moderno del Pentamerone, pubblicata nel 1989, De Simone ferma l'attenzione su un preciso evento storico, il processo del Risanamento iniziato cento anni prima, nel 1889, dopo cinque anni dal colera. Nella lunga trasformazione della città, tra fine Ottocento e inizio Novecento, lo sventramento dei quartieri bassi (Porto, Pendino, Zecca, Vicaria, Mercato) e di ben ottantadue fondaci, dove ancora "viva era la lingua del più vivo Cunto de li cuntï, e la separazione dell'antico borgo di Santa Lucia dal mare con il terrapieno e nuovi edifici, "nel moralissimo nome del Risanamento", costrinsero all'esodo sia gli abitanti ("figli della 'Nunziata', prostitute, scugnizzi, ladruncoli, femminelli, mendicanti veri e falsi, devoti della madonna dell'Arco, del Carmine, di Montevergine, ecc.") sia il dialetto che li univa, alla sparizione di quei luoghi e all'avvento della "luminosa e pulitissima civiltà del cemento 'armato"'12.

Il Risanamento, allora, secondo De Simone, segna una frattura tra il dialetto antico, quello secentesco, in parte ancora vivo e parlato negli anni Ottanta dell'Ottocento, e il dialetto "moderno", che nasce dalla diaspora e cresce in una nuova realtà, con l'inevitabile perdita di lessico del passato e creazione di lessico nuovo. Da qui, per rendere fruibile il Cunto di Basile,

${ }^{10}$ De Simone, La gatta Cenerentola, 14.

${ }^{11}$ De Simone, La gatta Cenerentola, 131.

${ }^{12}$ De Simone in Basile, Il Pentamerone, 1989, I, 11. 
la necessità di sostituire "vocaboli ed espressioni, che oggi risulterebbero incomprensibili, con un lessico che appartiene alla realtà odierna e quindi accessibile a tutti"13.

La preziosa Nota illustrativa "segna una svolta importante" nell'indagine sulla struttura del testo di Basile, "finalmente messa in relazione con il rosario"14. Infatti De Simone, nel rilevare "l'elemento mitico e magico-religioso delle favole", osserva che il Cunto ha "l'aspetto della recita di un vero e proprio 'rosario', composto di cinque misteri (= giornate), ognuno dei quali si articola mediante dieci grani, la cui somma complessiva forma il numero di cinquanta grani della mistica corona"15. Questa felice intuizione è stata ripresa e sviluppata da Salvatore Nigro, il quale ricorda che Basile in un'altra occasione "aveva contribuito con i suoi versi al Rosario di Maurizio Di Gregorio" e annota che nel Cunto "la recita rituale di una corona di cinquanta fiabe, dieci per ognuna delle cinque giornate" diviene celebrazione del "percorso di Zoza alla ricerca dell'ignoto: dal 'mistero gaudioso' a Vallepelosa, al settennale 'mistero doloroso' del pellegrinaggio di penitenza ed espiazione, al 'mistero glorioso' dell'incoronazione a Camporotondo"16.

Per De Simone il Cunto ha un "carattere teatrale" e lo dimostra confrontando il testo narrativo di Basile con la commedia La Lucilla costante (1623) del capuano Silvio Fiorillo, che fu messa in scena negli anni del concepimento del Pentamerone.

I monologhi della Lucilla [...] oltre a presentare molte analogie con i monologhi dei personaggi del Cunto, sono talvolta riconducibili a forme ritualizzate di linguaggio popolare, simile alle forme elencatorie del cantastorie, che s'incontrano frequentemente anche nell'opera di Basile. Così pure, nel Pentamerone, è frequentissimo l'uso di lamentazioni funebri o di 'repiti', in riferimento a una grande tradizione meridionale che, nel momento del cordoglio, si esprimeva con un vasto repertorio di stereotipi, di ritmi e di espressioni, aventi una carica di notevole teatralità ${ }^{17}$.

Da qui la scelta da parte di De Simone, nel rispetto della "vocazione teatrale" di Basile, di impiegare nella traduzione del Cunto "un particolare tipo di scrittura strutturata con ritmi, cadenze, scansioni ripetitive, che si

${ }^{13}$ De Simone in Basile, Il Pentamerone, 1989, I, 13-14.

${ }^{14}$ Nigro, "Il cunto de li cunti di Giovan Battista Basile", 888.

${ }^{15}$ De Simone in Basile, Il Pentamerone, 1989, I, 16.

${ }^{16}$ Nigro, "Il cunto de li cunti di Giovan Battista Basile", 878.

${ }^{17}$ De Simone in Basile, Il Pentamerone, 1989, I, 15. 
prestasse, insomma, a una recitazione agevole e fosse, nello stesso tempo, ricco di possibilità espressive"18.

Inoltre De Simone sottolinea il carnevale del Pentamerone, ottenuto con "un ironico impasto" di alto e basso, sublime e quotidiano, e con un divertito e divertente capovolgimento:

è l'ironia, infatti, ad abbassare spesso, nel corso della narrazione, il sublime a livello del quotidiano e ad innalzare il quotidiano alle altezze del sublime, riuscendo cos̀̀ a creare un continuo capovolgimento dei piani del reale e del fantastico. Attraverso tale gioco l'Ufficialità in tutti i suoi aspetti (letterario, politico, sociale, pseudo-moraleggiante) viene attaccata in chiave comica e grottesca, come in un raffinato Carnevale, dove la socierà riesce a ridere anche di se stessa ${ }^{19}$.

Ancora de Simone propone "un'altra chiave di lettura", quella della "contrapposizione" tra società femminile e società maschile, tra cultura femminile e cultura maschile. Da una parte, allora, le dieci donne (Zeza sciancata, Cecca storta, Meneca col gozzo, Tolla nasuta, Popa gobba, Antonella bavosa, Ciulla musuta, Paola strabica, Ciommetella tignosa, Iacova squacquarata) che narrano le favole, dall'altra i personaggi maschili (Fabiello e Iacovuccio, Cola Ambruoso e Marchionno, Giallaise e Cola Iacovo, Narduccio e Cicco Antuono) che al termine di ciascuna giornata riportano alla realtà storica nelle quattro egloghe a due voci (La coppella, La tintura, La stufa, L'uncino). E se le dieci vecchie danno vita a un mondo fiabesco, i personaggi maschili rappresentano un mondo senza fiabe, così la metastoria cede il posto alla storia, la favola alla realtà, il cunto all'egloga.

Per quanto riguarda le quattro egloghe De Simone adotta la linea della sostituzione "con composizioni centonizzate", capaci di dare ai lettori moderni "il senso che quei dialoghi avevano all'epoca di Basile". Con grande abilità, dunque, De Simone mette insieme, in nuovi componimenti centonizzati, frammenti di Velardiniello, Basile, Salvatore Di Giacomo, Ferdinando Russo, Giovanni Capurro, Totò ed il cantastorie Eugenio Pragliola detto Pucciariello ${ }^{20}$, senza seguire coordinate diacroniche e sincroniche:

Così, in luogo della prima egloga, vengono proposti alcuni versi di Velardiniello, poeta dialettale del XVI secolo, con cui vengono alternati versi di Salvatore Di Giacomo e di Ferdinando Russo. L'immagine che se

${ }^{18}$ De Simone in Basile, Il Pentamerone, 1989, I, 15.

${ }^{19}$ De Simone in Basile, Il Pentamerone, 1989, I, 16.

${ }^{20}$ Cfr. De Simone, "Viaggio in tram con Eugenio il cantastorie". 
ne ricava è quella di un canto lamentoso e struggente per la fine di un passato felice che non tornerà più. In sostituzione della seconda egloga, ad alcuni versi del Basile, che rimpiange la bella musica napoletana del '500, fanno da contrappunto i versi di un cantastorie popolare di Giugliano. Si tratta di Eugenio Pragliola, detto Pucciariello, che, fino a pochi anni orsono, nelle sue esibizioni quotidiane sui tram e sugli autobus della provincia di Napoli, improvvisava endecasillabi, ottonari e filastrocche di tipo giullaresco. Nei versi [...] egli rimpiange l'epoca della canzone napoletana con lo stesso spirito con cui il Basile si rammaricava che le belle 'villanelle' del passato siano state sostituite, al suo tempo, da canzoni 'in toscanese'. La terza egloga è stata sostituita da un centone formato da versi del Basile (Le nove Muse), da alcune formule ingiuriose tratte da La Gatta Cenerentola e da colorite espressioni desunte dal gergo dei 'femminelli'. In sostituzione della quarta egloga, è stato composto un centone con versi del su citato Pragliola, di Totò (da 'A livella), di Giovanni Capurro (da Totonno 'e Quagliarella) e dello stesso Basile. Con tali versi si tratta il tema della morte così come viene affrontato nella tradizione letteraria napoletana ${ }^{21}$.

Ma questa scelta di composizioni centonizzate non convince a lungo lo stesso De Simone, il quale nella citata edizione einaudiana della riscrittura del Cunto preferisce sostituire le quattro egloghe del Basile con quattro componimenti del poeta di strada Eugenio Pragliola.

\section{La doppia riscrittura, dialettale e italiana}

Negli anni Novanta, De Simone cura la pubblicazione delle Fiabe campane. $\mathrm{E}$ il risultato di una ricerca durata ben venti anni sui canti e sulle fiabe popolari, con la collaborazione di "alcuni giovani" che hanno svolto indagini in diverse aree, da Pomigliano (Vincenzo Grauso) all'area casertana (Gianni Gugliotta), dalla zona vesuviana (Antonio Orselli) all'Irpinia (Gennaro Vallifuoco), dove "meglio è conservata la memoria dell'immaginario collettivo della Campania" e dove Basile nel suo lungo soggiorno attinse "molto materiale" 22 per la sua raccolta.

La struttura della raccolta trae ispirazione da quanto avveniva fino agli anni Cinquanta in alcune aree della provincia di Caserta, dove la gente era solita riunirsi "di sera nei cortili intorno al fuoco", consumando "una rituale pietanza fatta di pasta asciutta e di castagne" e ascoltando alla fine "storie di Maghi, di Orchi, di Santi", raccontate da "narratori ufficiali". Tra

${ }^{21}$ De Simone in Basile, Il Pentamerone, 1989, I, 18-19.

22 Fiabe campane, I, xvii. 
le varie manifestazione, De Simone segnala quella che "si protraeva per dieci notti, dal giorno successivo all'Epifania (7 gennaio) fino alla vigilia di sant'Antonio Abate (16 gennaio) con la partecipazione dei più dotati narratori della zona" 23 .

Tra i novantanove racconti - "il centesimo racconto attende di essere scritto o narrato, di volta in volta, da ciascun lettore" 24 - si trovano anche 'E cunti d"a Cenerentola, con due versioni 'A gatta Cenerentola (registrata a Terzigno, in provincia di Napoli, nella frazione Boccia al Mauro, nel 1977) e Rospacennere (registrata a Villa di Briario, Caserta, nel 1974).

Nel 2002 appare la doppia riscrittura, in dialetto e in italiano: Il cunto de li cunti di Giambattista Basile nella riscrittura di Roberto De Simone. La genesi è raccontata dal Maestro:

L'idea venne a Giulio Einaudi nel novembre 1994, quando venne a Napoli per la presentazione delle mie Fiabe campane. Mi disse: "Maestro, mi devi fare una riscrittura di Basile in un napoletano comprensibile". Io gli chiesi: "Potete farmi un contratto?". E lui: "Probabilmente sarò morto quando avrai finito". È andata proprio così, e dunque il libro reca una dedica, A Giulio Einaudi25.

La Praefatio, scritta con il duplice registro linguistico - il latino in

23 Fiabe campane, I, xviii.

${ }^{24}$ Fiabe campane, I, xxvi.

${ }^{25} \mathrm{Cfr}$. l'intervista rilasciata a Titti Marrone, "La lingua musica". Sulle linee guida della doppia traduzione si veda, nella citata edizione, il risvolto di copertina, senzaltro ispirato dal riscrittore: "Roberto De Simone, nel rispetto dell'antico testo, ha semplificato la scrittura originaria, operando un'attenta eliminazione di complesse consonanti, sostituendo vocaboli oggi incomprensibili anche ai napoletani, cercando però di non alterare mai il ritmo basiliano e la sua musicalità sillabica, giungendo così a comporre un dialetto del tutto inventato, come specularmente risulta inventato quello originale del Basile. Infine, ha condotto la traduzione in italiano mantenendo sì la turgida costruzione del periodare barocco, ma, talvolta, per facilitare la scorrevolezza della lettura e agevolare la fruizione immediata dell'opera, ha provveduto a modificare la punteggiatura, riducendo l'eccessiva lunghezza dei periodi. Per quel che riguarda lo stile della scrittura, lungi dal tentare filologici compiacimenti letterari di falso antiquariato, De Simone ha impiegato l'italiano di oggi, pur riferendosi alla ricca teatralità dei modelli shakespeariani, o alla musicalità degli elenchi rabelaisiani. Del resto, è proprio il senso della teatralità del Basile, della sua ironia, delle sue allitterazioni, che De Simone ha cercato di trasporre nella sua riscrittura del testo". 
corsivo e l'italiano in tondo - è il cunto di Roberto De Simone, il quale, appropriatosi degli strumenti narrativi di Basile, opera in proprio, quale nuovo e moderno scrittore di cunti. Il riscrittore del Cunto, allora, crea un cunto alla maniera di Basile e lo colloca tatticamente sulla soglia del testo einaudiano, come Prefazione alla sua riscrittura.

L'elemento capitale del cunto di De Simone è il sogno. Infatti alla fine della Praefatio il lettore scopre, - assieme ai personaggi, con gli occhi dei personaggi - , che tutto vive in un sogno, il sogno di De Simone:

\section{BASILE}

Quindi, a quanto vedo, cavalier De Simone, ci troviamo nel vostro letto. Mi par di capire che stiamo vivendo in un vostro $\operatorname{sogno}^{26}$.

E qui il rovesciamento dei rapporti tra Basile e De Simone è totale: come De Simone ha vissuto a lungo nel sogno del Cunto di Basile, così ora Basile vive nel sogno del cunto di De Simone.

Il luogo della convocazione dei personaggi - si legge nella prosa latina - è l'antico e nobile palazzo dei marchesi di Battiloro, in Piedimonte Matese, dove De Simone sogna di riposare in un letto appartenente non a caso all'epoca del Basile:

In amoenissimo loco qui Piedimonte Matese nuncupatur est pervetusta, capax et spatiosa domus quae fuit olim marchionem Battilorae genti. (I, xiii)

La spalliera del letto è "tutta intagliata in legno dorato", arricchita con "un singolare stemma", nel quale "appariva un braccio la cui mano impugnava un martello, e il braccio era sollevato nell'atto di battere su un'incudine".

I convocati sono tutti "excellentissimi et prudentissimi viri" (I, xiii), Basile, Croce e De Simone. Con il registro linguistico alto del latino vengono esibiti i titoli dei singoli personaggi. Basile "equestri dignitate insignitus, Toronensis comes, Neapolitano ac Italico sermone eruditissimus, praefectus Montis Marani, sodalis Academiae Incautorum, comes Castri Rampae, praefectus Aversae iussu proregis Antonii Alvarez de Toledo, Albae ducis, praefectus Iuliani". Croce "splendidissimum lumen philosophiae, studii et litteris deditus, praeclarus rerum scriptor". De Simone "candidissima anima, artis musicae et scenicae Neapolitanae studiosissimus, idemque gradu equitis ornatus necnon Sanctae Caeciliae Academicus". A questi personaggi eccellenti si aggiunge, nell'ultima parte

${ }^{26}$ Il cunto de li cunti di Giambattista Basile nelle riscrittura di $R$. De Simone, I, xxxii. Le citazioni, relative a tale scritto, saranno indicate con il solo numero di pagina. 
del cunto, "un anonimo illetterato", Eugenio Pragliola, autore popolare dei quattro componimenti che De Simone colloca nell'edizione einaudiana al posto delle quattro egloghe del Basile.

Ad eccezione di De Simone, i personaggi sono tutti morti - Basile nel 1632, Croce nel 1952, morto è anche Pragliola - e ciascuno è stato condannato a una particolare pena nell'altro mondo, la cui generica geografia viene disegnata solo con il verticalismo alto-basso.

Basile, a causa delle "innegabili scurrilità" (I, xix) contenute nel Cunto, alloggia in una zona bassa dell'altro mondo ed è condannato a "leggere, in varie lingue, tutto ciò che hanno pubblicato i numerosi revisori del Cunto, le coglionate linguistiche, le correzioni al testo, le interpretazioni a vanvera..." (I, xviii), con l'esclusione di quanto ha scritto l'“eccelso", l'“illustre" Benedetto Croce. Inoltre Basile non si spiega perché Rabelais, che ha scritto un maggior numero "di porcherie", è in un'area più alta nella geografia dell'altro mondo.

Croce "alberga nel padiglione privilegato dei filosofi" e su di lui pesa la condanna di "leggere tutte le opere del De Simone", di "ascoltare la sua musica” (I, xxi). La sofferenza di Croce, allora, è grande e la pena è insopportabile, come spiega lo stesso personaggio filosofo:

Innanzitutto egli [De Simone] ha un pessimo rapporto con la Storia, un nebuloso concetto di letteratura, in cui accampa pretese demopsicologiche che, personalmente, ho sempre riguardate con sospetto. Per quel che concerne la musica, poco me ne intendevo anche quando ero in vita, e si immagini la tortura di sorbirmi suoni che nulla producono di estetico, ma che si attivano chiaramente fuori d'ogni processo attinente all'uomo e alla sua storia. (I, xxi)

Pragliola, poeta di strada che non sa leggere, è condannato alla lettura di tutti i libri di Croce, grazie all'aiuto di "un assistente ai morti analfabeti", con il prevedibile effetto apertamente dichiarato:

Mi sono fatto due palle, con rispetto parlando, quanto una mongolfiera. (I, xxix)

La pena di Eugenio addiziona la noia della lettura di Croce, il riso per non aver capito e il dolore per l'irrefrenabile riso:

quando sento leggere le cose che non si capiscono, le capisco a modo mio e mi scappa una risata a schiattafegato, che mi viene un dolore nei fianchi, e mi sgonfia la mongolfiera. (I, xxix)

A segnare marcatamente i personaggi sono le funzioni del corpo 
umano (come nelle Fiabe Campane, p. xcxiii)27, anzi le disfunzioni, in particolare quelle del basso ventre. Così il cavalier Basile è affetto da una "tosse stizzosa" (I, xiv), De Simone dallo "stimolo urinario, per allentamento della prostata" ( $\mathrm{I}, \mathrm{xv})$, Croce da "un peteggiare involontario, ereditato dall'aerofagia patita prima di morire" (I, xx).

Il motivo della convocazione è, per l'appunto, la recentissima pubblicazione presso Einaudi del Cunto de li Cunti di Basile:

Conventus causa fuit recentissima editio libri a Basile compositi, cui nomen est Il Cunto de li Cunti, typis librariae officinae Einaudi impressi. (I, xiii)

L'incontro avviene nel 2002 ("anno bis millesimo et altero p. Ch. n."), nell'ottavo giorno prima delle Calende di marzo, cioè il 21 febbraio ("ante diem octavum Kalendas Martias"), a mezzanotte ("media nocte").

Più avanti il testo latino segnala lo scorrere delle ore e il suono che annuncia la fine della notte:

Itaque ille (De Simone) foras egreditur dum sonitus quartae vigliae ineuntis in aera effunditur. Paulo post (horrendum auditu!') fortissimus ventris crepitus aures Johannis Baptistae Basile percutit simulque vehementi flatu micant omnium lampadum flammae quae in domo sunt. Hic in conclave intrat Benedictus Croce: tacitus, summissa cervice, equitem Basile salutat qui invicem graviter et apte respondet nutans ac molliter tussiens. (I, xix-xx)

Mancano ulteriori indicazioni sul tempo della vicenda, ma l'alba entra in scena per allusione, grazie allo scambio di battute tra De Simone che, "tormentato dall'assillo urinario", chiede scusa perché deve lasciare la compagnia, e Basile che lo ferma per un attimo, dicendogli: "Sapevate che l'atto del pisciare è antica e magica rappresentazione dell'alba che sorge?" (I, xxiii).

La chiave carnevalesca del cunto è offerta al lettore sin dall'inizio, nel contrasto tra il personaggio De Simone, che indossa "uno smoking, un abito da cerimonia", "una divisa atta ad alta circostanza", come quella dell'incontro con Basile, e il personaggio Basile, il quale dichiara che "l'abito stravagante" indossato dal suo interlocutore lo ha indotto a scambiarlo "per un servo in capriccioso abbigliamento carnevalesco", con "una ridicolosa livrea di carnevale" (I, xiv).

${ }^{27}$ Così avviene anche nei racconti popolari. Cfr. l'Introduzione di De Simone alle Fiabe campane, I, xxiii. 
Nel gioco del contrasto, Basile accusa De Simone di essere "il ricopiatore, il trascrittore, il rifacitore" e De Simone che si difende:

Dica pure il riscrittore, il curatore, il traduttore, se le aggrada (I, xv).

Nel sogno di De Simone, Basile definisce il Cunto de li cunti "una gran minestra maritata, un cocktail culinario in cui c'è di tutto e di più: la carne del Boccacciio, le noglie della tradizione, le verzure del Marino, le uova del Tasso, il sale della mia zucca e il pepe della lingua napolitana" (I, XV), e De Simone che si schermisce: "Conosco bene la minestra maritata, ma non mi sarei permesso una similitudine tanto irriverente" (I, xv-xvi).

Il personaggio Basile appare senza peli sulla lingua e, nello spingere De Simone a mettere da parte l'indiscrezione e ad alzare il sipario sui peli del Cunto, lo avverte che anch'egli conosce bene tutti i peli della sua Gatta.

Siamo qui di fronte a due opere, - il Cunto de li cunti di Basile e $\mathrm{La}$ gatta Cenerentola, che è il cunto de li cunti della gatta Cenerentola di De Simone -, rappresentate come parti del corpo, come orifizi anali abitati da peli. E l'insistenza sui peli trasforma quelle parti del corpo in vere e proprie scene boscherecce:

BASILE

Ohibò, adesso mi prende per il culo, et quod sermo in stercus decidit, se è proprio lì che vuole andare a parare, non faccia complimenti, si accomodi, visto che s'è adoprato a contarmi i peli del buco.

DE SIMONE

Veramente io...

BASILE

Non lo neghi, ché se non me li ha contati, e parlo del buco letterario, non può vantare una piena conoscenza del mio Cunto.

DE SimONE

Non posso negarlo, equidem.

BASILE

Ma stia pur certo che le ho ricambiato l'indiscrezione, e anch'io conosco i peli della sua Gatta. $\quad(\mathrm{I}, \mathrm{xvi})$

L'alzata di sipario sui peli del buco - il buco letterario - nel cunto di De Simone rimanda a un'altra alzata di sipario, che il lettore della riscrittura del Cunto de li cunti trova, guarda caso, nella 'ntroduzzione dello stes- 
so Basile, il quale racconta che c'era una volta il re di Vallepelosa, che per far ridere la malinconica figlia di nome Zoza tentò un'ultima prova e fece costruire una fontana d'olio davanti al palazzo reale, "co disegno che, sghizzanno l'uoglio a lo passare de la gente, che pe' chella strada facevano lo vae-viene comm'a fformicole, pe' non serógnere li vestite, averriano fatto zumpe de grillo, sbauze de crapio, corze de lepre, e sciulianno o tozzannose chisto e chillo, potesse succedere cosa pe' la quale se scoppiasse a ridere la Principessa" (I, 6). Una vecchia cercò di riempire d'olio "n’agliaro" (contenitore per liquidi), ma un "birbantiello de paggio" tirò un sasso "così a pilo" (a segno) da mandarlo in frantumi. A questo punto la vecchietta, "che non aveva pilo a la lengua", dapprima gli scaricò addosso una serie di maleparole e poi, "aizata la tela de l'apparato, fece vedere la scena boscareccia" (I, 8). Fu tale l'orrore che "il vello pubico di satiressa" 28 svegliò in chi la guardava, da ricordare il corno che nel Pastor fido del Guarini sveglia i cacciatori del cinghiale ${ }^{29}$. Allo stesso modo, quella scena boschereccia della vecchia fu come il corno che svegliò la principessa Zoza dalla sua malinconia.

L'elenco dei peli del Cunto de li cunti di Basile procede in modo serrato nel cunto di De Simone:

La sua concisione del comico nel dilagare dei cataloghi farebbe pensare a Rabelais. (I, xvi)

L'impostazione letteraria del Cunto deriva dal Decamerone. (I, xvii)

E la magia? Agrippa, Paracelso o Giordano Bruno? (I, xvii)

[La lingua del Cunto è] composta da un crudo napoletano a volte italianizzato, da un cotto italiano napoletanizzato, ma soprattutto da salati modi di dire [...]. (I, xviii)

L'arrivo in scena del personaggio Benedetto Croce, in ritardo rispetto alla convocazione, è salutato da frizzi salaci e vari rumori. Si va dalle battute di Basile e De Simone:

BASILE

Ma tornando al Croce, son stupito che non sia ancora giunto. Potrei quasi dire che è un filosofo in ritardo. (I, xix)

28 Nigro, "Il cunto de li cunti di Giovan Battista Basile", 875.

${ }^{29}$ Guarini, Il pastor fido, I, I, 3-4, in Il teatro italiano, II, 737. 


\section{DE Simone}

E una battuta? Che forse si sia perso?

\section{BASILE}

È probabile, egli è un vichiano puro, e i Vichi del Signore sono infiniti, altrimenti dovrei ritenermi già offeso [per il ritardo]. (I, xix)

ai rumori incalzanti dello scroscio urinario di De Simone, della tosse stizzosa di Basile, del peteggiare fortissimo dello stesso Croce, il quale per la sua ventosità anale viene rappresentato parodicamente come portatore di "ventosità filosofiche" ( $\mathrm{I}, \mathrm{xx})$.

Da questo momento il personaggio Basile diviene il giudice della contesa tra Croce e De Simone, tra il revisore del Cunto de li cunt 30 e il riscrittore. Nella contesa, Croce giudica "pessima", sul piano storico e letterario, sia la riscrittura in napoletano fatta da De Simone, a causa delle alterazioni del dialetto secentesco e dell'inserimento di poetastri di strada, sia la riscrittura in italiano a causa dei neologismi e delle basse volgarità:

Pensi lei [rivolto a Basile] che egli [De Simone] si è concesso la libertà di alterare il testo da lei composto, per renderlo, a suo dire, più comprensibile agli stessi napoletani. La traduzione italiana è indigestamente farcita di neologismi e di grasse volgarità che possono strappare il riso ma non una stima culturale. Al testo originale, laddove si espone il catalogo dei giochi e dei canti da lei compilato, egli ha aggiunto giochi e canti di tradizione napoletana più recente, in special modo all'inizio della quarta e della quinta giornata, in cui ha osato introdurre addirittura il singulto di poetastri di un cosiddetto stile 'rap', né ho trovato il senso di questo barbarismo nel Devoto-Oli. (I, xxi-xxii)

E ancora Croce boccia perentoriamente la sostituzione delle egloghe del Basile e accusa De Simone di aver fatto uso di "tropi" troppo licenziosi:

${ }^{30}$ A fine Ottocento Croce pubblicò solo le prime due giornate dell'opera: Lo cunto de li cunti. Il Pentamerone. A inizio Novecento fornì una traduzione italiana dell'intero testo e lo annotò: Il Pentamerone, ossia La fiaba delle fiabe. Seguì un opuscolo con correzioni, integrazioni e notizie sulle traduzioni inglesi e tedesche: Appendice all'edizione italiana. Il Pentamerone crociano è stato ristampato (Roma-Bari, Laterza, 1974, 3 voll.) con la prefazione di Italo Calvino ("La mappa delle metafore", poi in Calvino, Sulla fiaba, 129-146). Gli studi di Croce sul Basile sono stati raccolti in Saggi, 1-105 (Giambattista Basile e il "Cunto de li cunti», 1891) e in Storia dell'età barocca, 451-470 (Giambattista Basile e l'elaborazione artistica delle fiabe popolari, 1925). 
Ha del tutto sostituito le egloghe originali, interpolate tra una giornata e l'altra, con delle illetterate composizioni di un giullare di strada, tale Eugenio, che, in vita, si produceva in estemporanei monorimi presso gli stazionamenti dei tram diretti in provincia. (I, xxii)

Ho espresso semplicemente il mio punto di vista, obiettando sulla liceità dei tropi introdotti, a mio giudizio troppo audaci e impertinenti. (I, xxiii)

A conti fatti, dopo che De Simone ha contato i peli del Cunto di Basile, Croce conta i peli del Cunto riscritto da De Simone.

Infine, nella contesa Croce-De Simone, il personaggio De Simone si mette a sua volta a contare i peli del Cunto rivisto da Croce e cerca il consenso del giudice Basile:

se ella ha contato i miei peli, io le ricambierò la cortesia. Ed è inutile che peteggi, adesso! Cavalier Basile, ascolti in qual modo l'eccelso critico si pronuncia su alcuni aspetti del suo Cunto. Leggo dalla prefazione al Pentamerone del 1925: "Mi sono condotto con piena libertà di rifacimento verso la sintassi, che nel Basile è difettosa e spesse volte pessima". (I, xxiv)

E Croce interviene nel tentativo di esporre e far valere le sue ragioni:

Ma ho aggiunto: "forse principalmente perché l'opera fu stampata ancora incondita e in molte parti quasi in abbozzo". (I, xxiv)

A questo punto Basile, colpito nel suo punto debole, smette gli abiti di giudice, si trasforma in duellante e attacca Croce, facendo proprie le accuse mosse da De Simone, il quale a sua volta, da duellante contro Croce, si trasforma in giudice e paciere della contesa Basile-Croce:

BASILE [rivolto a Croce]

Ma chi glielo ha detto? Chi glielo ha raccontato? Che cazzo... pardon!

De Simone

Si calmi, non tossisca, e lei non peteggi!

BASII.E

Cavalier Croce, ella ha mentito!

CROCE

Non ho mentito. 


\section{BASILE}

Ella mente di non aver mentito!

\section{DE Simone}

Siamo a un duello! Suvvia, signori, ogni verità è menzogna. Cavalier Basile, il Croce ha avanzato solo un'ipotesi.

\section{BASILE}

Ma ha definito pessima la mia sintassi. Che ne sa, lui? Io mi sono ispirato, in taluni momenti, a una costruzione non meditata, di sapore estemporaneo, di stile orale. Assolutamente non grammatichese, pur se letteraria e prodotta intenzionalmente.

\section{CROCE}

Ma ciò che è scritto rimane scritto. Ne convenga.

\section{DE SiMONE}

Il Cunto è stato indirizzato a li 'peccerille', e i piccerilli ascoltano, ma non sanno leggere. (I, xxiv-xxv)

Da una parte, dunque, il personaggio Basile che difende la propria scrittura, che ha sì una dimensione letteraria, ma tende a riprodurre la lingua orale, dall'altra Croce che difende la separazione tra lingua scritta e lingua parlata. Infine il giudice De Simone che interviene contro il revisore Croce e a favore della necessaria dimensione orale della scrittura di Basile, dal momento che i destinatari del Cunto sono i piccerilli, un pubblico di uditori più che di lettori.

Superato lo scontro tra Basile e Croce, si ritorna al duello tra Croce e De Simone sulla questione della sostituzione delle egloghe, operata dal Maestro, con le "tirate di strada" di Eugenio Pragliola. In questo caso Croce è dalla parte di Basile:

Non le sembra irriverente, cavalier De Simone, accostare un anonimo illetterato a un erudito e geniale scrittore qual è il Basile? (I, xxv)

E Croce bolla tutto in questo modo:

Metodi e teorie sorti ai tempi del fanatismo per la linguistica comparata e per la sua genealogia dei linguaggi e per la congiunta ricerca della prima scaturigine storica del linguaggio. (I, xxviii)

In questo caso il personaggio Croce, delineato dallo scrittore De Simone nella Prefatio alla riscrittura del Cunto di Basile, sta citando Croce 
autore, e precisamente uno stralcio breve tratto, guarda caso, dalla Prefazione (Giambattista Basile e l'elaborazione artistica delle fiabe popolari) di Croce all'edizione laterziana (1925) del Pentamerone ossia la Fiaba delle fiabe di Giambattista Basile ${ }^{3 !}$.

La difesa di De Simone si fonda su alcuni punti: a) le egloghe di Basile "non trovano più alcun riferimento con la realtà attuale di Napoli"; b) la "soppressione" non "pregiudica il valore sostanziale del Cunto"; c) la sostituzione con i componimenti di Eugenio Pragliola, conosciuto intorno al 1965, è legata anche al fatto che egli, pur essendo nato in Brasile, "è della stessa patria” di Basile, Giugliano, paese originario di suo padre (I, xxv).

Il riso è elemento costante e naturalmente conclusivo del cunto di De Simone. Infatti nelle ultime battute siamo di fronte a una somma di riso. Il primo addendo è fornito dal poeta di strada Eugenio che dichiara di ridere alla lettura delle opere di Croce perché, non comprendendole, le capisce a modo suo. Il secondo addendo è dato da De Simone che rileva nel Basile "l'intenzione di far ridere il pubblico" (I, xxx). Il terzo addendo è esibito ancora da Eugenio, il quale ribadisce che, quando era vivo, anch'egli "modestamente" faceva la stessa cosa. Infine il quarto addendo viene nientedimeno che dal filosofo e revisore Croce, il quale con convinzione confessa: "Avrei tanto voluto scrivere dei testi comici anch'io" ( $I, \mathrm{xxx}$ ), ma poi si è arrestato per non imbrattare l'immagine dell' "illustre filosofo" con quella dell'“autore di battute e di pochade" (I, xxxi). Di rincalzo Basile suggerisce, col senno di poi, che "avrebbe potuto coprirsi con uno pseudonimo", coniando l'anagramma di Benedetto Croce, come "ad esempio: Cerotto Bencede" (I, xxxi).

In vena di confidenze, il personaggio Croce racconta che, dopo aver difeso in tribunale Eduardo Scarpetta ${ }^{32}$ per La figlia di Iorio (Napoli, Morano, 1906), rappresentata al Teatro Mercadante di Napoli (3 dicembre 1904) ${ }^{33}$ e accusata di plagio dell'opera di D'Annunzio Il figlio di Iorio ${ }^{34}$, propose all'autore-attore di mettere in scena sotto il nome di Scarpetta la commedia Il medico dei pazzi, spacciata ora come commedia crociana. La parodia di Croce raggiunge qui il punto più alto, mostrando il re nudo,

${ }^{31}$ In nota De Simone indica anche le pp. xxxi-xxxii.

${ }^{32}$ Sui rapporti di amicizia tra il filosofo e l'autore-attore cfr. Eduardo Scarpetta, Cinquantanni di palcoscenico; Maria Scarpetta, Felice Sciosciammocca.

${ }^{33} \mathrm{Cfr}$. l'anonima recensione in Il Pungolo, 5 dicembre 1904.

${ }^{34}$ Cfr. AA.VV., "Pel Figlio di Iorio". Processo D'Annunzio-Scarpetta. 
additando il pubblico filosofo illustre come privato autore di un testo comico di grande successo, accostando con provocatoria irriverenza filosofia e pazzia.

Al termine della Praefatio, il lettore trova un'avvertenza: "Il testo latino fu redatto da Giambattista Vico all'insaputa di Benedetto Croce" (I, xxxiii). È l'ultimo colpo di scena nel teatrale cunto di De Simone. Infatti il lettore scopre solo alla fine che, secondo la finzione narrativa, la Praefatio scritta da De Simone viene attribuita per la parte latina a Giambattista Vico. Un cunto, dunque, a due mani e di due autori - Vico per la parte latina e De Simone per la parte italiana —, così come De Simone nel gioco della finzione vuol far credere al lettore.

Al lettore, a mio avviso, non rimane altro che stare al gioco, per cogliere un'ultima staffilata parodica. Infatti, rileggendo ora il cunto, Vico e De Simone, i due autori, appaiono in perfetta sintonia e operano una salutare sinergia:

Primi convenere duo equites Baptista Basile et Robertus De Simone, qui post fervidas salutationes pergrato animo accubuere in convivio tantis hospitibus parato. Hic initium habuit inter eos disputatio de permultis variisque ineptiis sed praecipue de anilibus fabellis ad pueriles animos oblectandos aptis. Huiuscemodi circiter fere verba doctissimi colloquii, quae Italico sermone hic referuntur. (I, xiii)

Vico nella prosa latina racconta al passato e in terza persona il sogno di De Simone e De Simone a sua volta nella prosa italiana racconta al presente il dialogo tra i personaggi del sogno. Vico svolge il ruolo di narratore onnisciente in quanto conosce perfettamente il sogno, dal momento che, a ben vedere, è nel sogno di De Simone, come testimone-spia di quello che avviene, per cui anche questo secondo narratore è nel contempo personaggio. Non a caso c'è un'indicazione precisa nel testo, che adesso può essere recuperata, là dove Croce, scusandosi per il ritardo del suo arrivo, afferma di aver avuto l'impressione "di scorgere il gran Giambattista" (I, xx), il suo grande Giambattista Vico, lungo la strada e nel buio.

Sorpresa delle sorprese, nel carnevale della Praefatio per una volta il togato e serio Vico di Croce - il Vico del filosofo abruzzese indicato da Basile come "un vichiano puro" (I, xix) -, all'insaputa di Croce diventa il Vico familiare e scherzoso di De Simone, anzi il Vico che scrive una parodia di Croce a due mani con De Simone. È davvero il caso di dire, con quella battuta saporita che nel cunto di De Simone è attribuita a Basile, "i Vichi del Signore", ovvero i Vichi della letteratura, "sono infiniti" (I, xix).

Agli storici della letteratura, che dovranno dare sistemazione critica a 
questo primo secolo del nuovo millennio, possiamo lasciare un appunto, che potrebbe alla fine essere utile. Si tenga presente la coincidenza, nel 2002, tra la pubblicazione del cunto di De Simone e il cinquantenario della morte di Croce (finito nel 1952). Capita, allora, nell'anno 2002 che, mentre in tutta Italia e all'estero si moltiplicano i convegni per ricordare e celebrare Croce, nel coro si leva la voce discordante di De Simone, il quale mette in scena la parodia di Croce.

Nell'anno di Croce, dunque, il cunto di De Simone rappresenta il carnevale di Croce e dell'egemonia che ha esercitato nella cultura napoletana ed europea. E il riscrittore De Simone appare per quello che è, un post-crociano, anzi un anti-crociano, alla ricerca tra il passato e il presente di una nuova scrittura con cui tessere il cunto di Napoli che si libera dall'ultimo padre.

\section{UNIVERSITA DEgli STUdi di Napoli Federico II}

\section{OPERE CITATE}

AA.VV., "Pel Figlio di Iorio". Processo D’Annunzio-Scarpetta. Napoli: Morano, 1908.

Anonimo. Il Pungolo. 5 dicembre 1904.

Basile, Giambattista. Lo cunto de li cunti. Pentamerone (testo conforme alla prima stampa del 1634-36), I. Napoli: s.n.t. (Trani, V. Vecchi), 1891.

- Il cunto de li cunti di Giambattista Basile nella riscrittura di Roberto De Simone. Note di C. De Iudicibus, illustrazioni di G. Vallifuoco. Torino: Einaudi, 2002.

. Il Pentamerone, ossia La fiaba delle fiabe, tradotta dall'antico dialetto napoletano e corredata di note storiche. 2 voll. Bari: Laterza, 1925.

Il Pentamerone, ossia La fiaba delle fiabe, tradotta dall'antico dialetto napoletano e corredata di introduzione e note storiche di B. Croce, prefazione di I. Calvino. 3 voll. Roma-Bari: Laterza, 1974.

_. Il Pentamerone ovvero Lo cunto de li cunti trattenimento de piccerille. Napoli: Il Mattino, 1989.

Calvino, Italo. Sulla fiaba. A cura di M. Lavagetto. Torino: Einaudi, 1988.

Croce, Benedetto. Appendice alledizione italiana del "Cunto de li cunti" di G. B. Basile. Bari: Laterza, 1939.

-. Saggi sulla letteratura italiana del Seicento. Bari: Laterza, 1962.

—. Storia dell'età barocca in Italia. Bari: Laterza, 1967.

De Simone, Roberto. La gatta Cenerentola. Torino: Einaudi, 1977.

"Linguaggio e tradizione nel teatro di Eduardo" pp. 97-154 in L'arte della commedia. Atti del Convegno di studi sulla drammaturgia di Eduardo (Roma, 21 settembre 1988). A cura di A. Ottai - P. Quarenghi. Roma: Bulzoni, 1990.

- Lopera buffa del giovedì santo. Torino: Einaudi, 1999. 
—. "Viaggio in tram con Eugenio il cantastorie", Il Mattino (15 agosto 2005): 20-21.

Di Stefano, Paolo. "Ma ora liberiamoci di Eduardo" Corriere della Sera (19 gennaio 2006): 58.

Fiabe campane. I novantanove racconti delle dieci notti. A cura di R. De Simone, commento e note di U. Vuoso, ventidue illustrazioni originali di G. Vallifuoco. voll. 2.Torino: Einaudi, 1994.

Guarini, Battisti. Il pastor fido in Il teatro italiano, II. Teatro del Cinquecento. La tragedia del Cinquecento. A cura di M. Ariani, Torino, Einaudi, 1977. 723-947. Marrone, Titti. "La lingua musica" Il Mattino (2 ottobre 2002): 21.

Nigro, Salvatore S. "Il cunto de li cunti di Giovan Battista Basile" in Letteratura italiana. Le opere, dir. A. Asor Rosa, II. Dal Cinquecento al Settecento. Torino: Einaudi, 1993. 867-891.

Scarpetta, Eduardo. Cinquantanni di palcoscenico. Memorie. Introduzione di R. Carpentieri, prefazione di B. Croce, Milano, Savelli, 1982.

Scarpetta, Maria. Felice Sciosciammocca, mio padre. Napoli: Morano, 1949. 